\title{
A Fog Computing Simulation Approach Adopting the Implementation Science and IoT Wearable Devices to Support Predictions in Healthcare Environments - Extended Abstract - CTDSI/CTCCSI 2021
}

\author{
Thiago G. Thomé ${ }^{1}$, Victor Ströele ${ }^{1}$, Mario A. R. Dantas ${ }^{1}$, Hélady Pinheiro ${ }^{1}$ \\ ${ }^{1}$ Federal University of Juiz de Fora (UFJF) \\ Juiz de Fora - MG - Brazil \\ \{thiagogoldoni,victor.stroele, mario.dantas\}eice.ufjf.br, heladysandersegmail.com
}

\begin{abstract}
In the Covid-19 pandemic, it was already possible to obtain recommendations to assist in the control of contamination. In this study, we considered the implementation science concept in a simulation effort based on changes in prevention behaviors. We also considered the use of an information system and wearable IoT devices for monitoring people in environments where social isolation is complex. We conceived four scenarios with different approaches, where health data of the simulated agents were collected for monitoring and providing predictions. Agents with more preventive habits got contamination rates of $12.11 \%$ against the worst scenario, with $77.00 \%$.
\end{abstract}

\section{Introduction}

In the context of Covid-19 pandemic, some environments, such as nursing homes and hospitals, social distance is more complex, requiring the use of other prevention measures. Since Sars-CoV-2 can be transmitted during the incubation phase, people who live or visit such environments should be closely monitored [Lauer et al. 2020]. The use of wearable IoT devices is one way to approach it, allowing the collection of data such as heart rate, blood pressure, blood oxygenation level and body temperature.

An important challenge of this proposal is to provide the information collected to the stakeholders, who may be health professionals or relatives of those being monitored. To assist in the development of an architecture capable of improving the transfer and processing of the collected data, some concepts are of great value.

Filtering and cleaning methods are important due to the large volume of collected data in order to delete redundant information and shrink the total amount of data. As a result, a fog-cloud cooperative scheme is proposed to distribute part or all of the operations that would otherwise be concentrated in a single platform [Salah et al. 2020].

In addition, the development of a information system is extremely important. Among its main duties, the most important are data management and making predictions for monitored people. Due to handling extremely sensitive information, it must be developed following software engineering standards. Thus, ensuring the quality of the architecture and minimizing problems. It is important to emphasize the importance of ensuring security for the data collected and different access profiles to the system to ensure the privacy of those being monitored. 
One of the complexities involved in this study was applying a computational proposal that uses IoT paradigms to explain how the virus spreads in environments. Finally, our research question is: How to conceive a computational proposal adopting the IoT paradigm to easily demonstrate the indoor Covid-19 contamination? [Thomé et al. 2021]

\section{Materials and Methods}

The search for databases that fulfill all of the desired characteristics is difficult due to the fact that the health data collected by IoT devices is from the users themselves, and they prefer not to make the data public. Simulated environments were used to combine the Sars-CoV-2 scenario, IoT, and environments where social distance is difficult.

Using a simulator enables to picture our IoT paradigm proposal being implemented, and the Siafu was used to achieve these goals [NEC 2007]. Siafu is a simulator that allows managing environment characteristics, agent actions and the overall context. We considered a simulation model as an approach to tackle challenges related to research question. The scope includes from the data collection from simulated agents to the computational environment responsible for providing the health predictions. The proposed simulation model is shown in Figure 1.

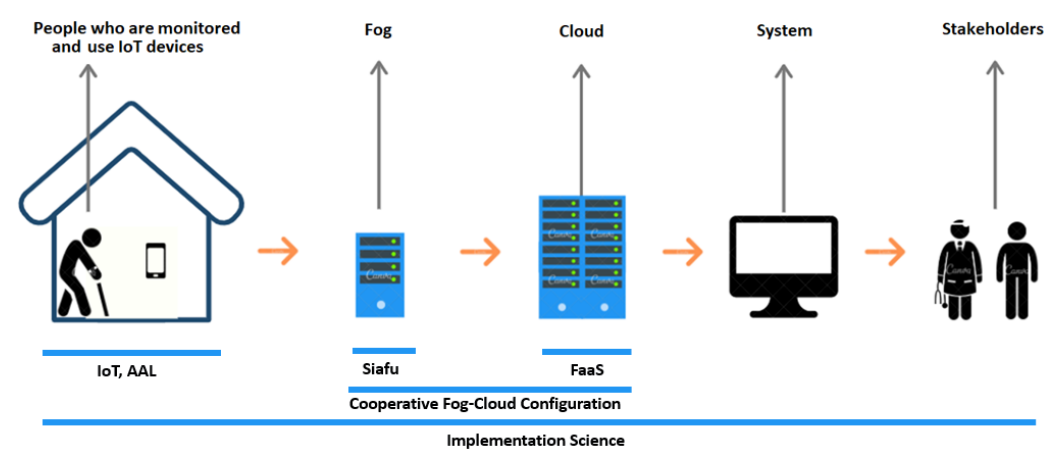

Figure 1. Simulation model

Only the simulation via Siafu, the creation of health data and the mobile application were implemented for a preliminary assessment. The environment, rooms, and individual agent characteristics were all created from the beginning. In this virtual setting, which is a nursing home with 18 agents, social distance is complicated.

\section{Proposed Approach, Experimental Environment and Results}

In the simulated model, many customized features of the agents were applied, as well as different behaviors before and after the knowing of the first case. Following the studies of [Liu et al. 2020] and [Lauer et al. 2020], the simulation's asymptomatic patient rate was increased to $30 \%$. The protection values for the use of protective equipment were in accordance with [Chu et al. 2020] meta-analysis. The transmission models developed in the simulation were made in conjunction with researchers in the health field. Tables 1 defines the four environments that were created to enable comparisons of the agents' behaviors and practices. An important difference is that in environments 1 and 2, the agents with symptoms are isolated, contrary to what happens in the others.

During the testing stage, each simulation was subjected to 50 executions. The SX-Aurora TSUBASA computer from NEC was used for all of them [NEC 2020]. Table 
Table 1. Habits and preventive measures considering the first case revelation

\begin{tabular}{lllll}
\hline Period of time about the 1st case revelation & \multicolumn{2}{l}{ Environments 1 and 3 } & \multicolumn{2}{l}{ Environments 2 and 4 } \\
\hline & Before & After & Before & After \\
\hline Use of PFF2/N95 masks & $0 \%$ & $100 \%$ & $90 \%$ & $100 \%$ \\
Use of other kind of masks & $50 \%$ & $0 \%$ & $10 \%$ & $0 \%$ \\
Use of eye protection & $0 \%$ & $90 \%$ & $50 \%$ & $90 \%$ \\
Average increase in interpersonal distance & $0 \mathrm{~m}$ & $1.5 \mathrm{~m}$ & $1.0 \mathrm{~m}$ & $1.5 \mathrm{~m}$ \\
\hline
\end{tabular}

2 lists some of the study's main points of interest. The average values of contaminated agents before and after the announcement of the start of contamination are among them.

Table 2. Contaminated agents in all simulation environments

\begin{tabular}{lrrrc}
\hline Situation & \multicolumn{4}{c}{ Contaminated Agents in Environments } \\
\hline Environment & \multicolumn{1}{c}{ 1 } & \multicolumn{1}{c}{2} & \multicolumn{1}{c}{3} & \multicolumn{1}{c}{} \\
\hline Before first case revelation & $65,22 \%$ & $11,44 \%$ & $61,56 \%$ & $11,00 \%$ \\
After first case revelation & $2,67 \%$ & $0,67 \%$ & $15,44 \%$ & $14,89 \%$ \\
Total & $67,89 \%$ & $12,11 \%$ & $77,00 \%$ & $25,89 \%$ \\
\hline
\end{tabular}

\section{Conclusions and Future Works}

This research work clearly demonstrates the importance of isolation and prevention habits in the Covid-19 pandemic. The proposal has efforts which we will implement to provide a more broad vision of scenarios, considering a large digital storage and data retrieval.

\section{References}

Chu, D. K., Akl, E. A., Duda, S., Solo, K., Yaacoub, S., and Schünemann, H. J. (2020). Physical distancing, face masks, and eye protection to prevent person-to-person transmission of sars-cov-2 and covid-19: a systematic review and meta-analysis. volume 395, pages 1973-1987. The Lancet.

Lauer, S. A., Grantz, K. H., Bi, Q., Jones, F. K., Zheng, Q., Meredith, H. R., Azman, A. S., Reich, N. G., and Lessler, J. (2020). The incubation period of coronavirus disease 2019 (covid-19) from publicly reported confirmed cases: Estimation and application. volume 172, pages 577-582. Annals of Internal Medicine.

Liu, Y., Yan, L. M., Wan, L., Xiang, T. X., Le, A., Liu, J. M., Peiris, M., Poon, L., and Zhang, W. (2020). Viral dynamics in mild and severe cases of covid-19. pages 656-657. Lancet Infect Dis.

NEC (2007). Siafu an open source context simulator. http://siafusimulator.org/ (accessed on $07 / 2020$ ).

NEC (2020). Sx-aurora tsubasa. https://www.nec.com/en/global/solutions/

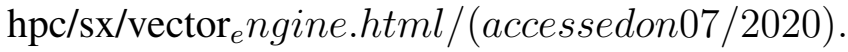

Salah, F. A., Desprez, F., and Lebre, A. (2020). An overview of service placement problem in fog and edge computing. volume 53. Association for Computing Machinery.

Thomé, T. G., Ströele, V., Pinheiro, H., and Dantas, M. A. R. (2021). A fog computing simulation approach adopting the implementation science and iot wearable devices to support predictions in healthcare environments. In Advanced Information Networking and Applications, volume 226. Springer, Cham. 